\title{
Concurrent Chemoradiotherapy Combined With Nimotuzumab in Stage III-IVa Nasopharyngeal Carcinoma: a Retrospective Analysis
}

\section{Zhuochen Cai}

Sun Yat-sen University Cancer Center

\section{Dongni Chen}

Southern Medical University

\section{Wenze Qiu}

Guangzhou Medical University

\section{Chixiong Liang}

Sun Yat-sen University Cancer Center

\section{Yingying Huang}

Sun Yat-sen University Cancer Center

Jiayu Zhou

Sun Yat-sen University Cancer Center

\section{Zejiang Zhan}

Sun Yat-sen University Cancer Center

\section{Yanqun Xiang}

Sun Yat-sen University Cancer Center

\section{Xiang Guo}

Sun Yat-sen University Cancer Center

Xing Lv ( $D$ lvxing@sysucc.org.cn )

Sun Yat-sen University Cancer Center https://orcid.org/0000-0003-3125-6853

\section{Research Article}

Keywords: Nasopharyngeal carcinoma, Nimotuzumab, Concurrent chemoradiotherapy, Survival, Prediction model

Posted Date: February 7th, 2022

DOI: https://doi.org/10.21203/rs.3.rs-1316434/v1

License: (a) (i) This work is licensed under a Creative Commons Attribution 4.0 International License. Read Full License 


\section{Abstract}

Purpose: The efficacy and safety of nimotuzumab (NTZ) added to concurrent chemoradiotherapy (CCRT) was investigated in patients with stage III-IVa nasopharyngeal carcinoma (NPC).

Methods: Patients with stage III-IVa NPC treated with CCRT, with or without NTZ, were screened between January 2015 and December 2017. Overall survival (OS), progression-free survival (PFS), locoregional recurrence-free survival (LRFS), and distant metastasis-free survival (DMFS) were compared between these groups. Propensity score matching (PSM) was applied to reduce the selection bias. Nomogram models were developed to predict survival of CCRT with or without NTZ.

Results: A total of 426 patients were included after PSM, with 213 patients in each regimen. Compared with NPC patients receiving CCRT alone, patients who received NTZ plus CCRT treatment had significantly better OS (5-year OS, $76.1 \%$ vs. $72.3 \%, P=0.004$ ), PFS (5-year PFS, $73.2 \%$ vs. $69.0 \%, P=$ 0.002 ), and LRFS (5-year LRFS, $73.2 \%$ vs. $69.0 \%, P=0.028$ ). A multivariate Cox regression analysis demonstrated that, compared with receiving CCRT alone, NTZ plus CCRT was an independently positive factor for OS, PFS, and LRFS. In addition, no significant difference was observed in the major toxicities among the two treatments (all $P>0.05$ ). Nomogram models that identified significant prognostic factors were produced.

Conclusion: The present study suggested the NTZ plus CCRT treatment presented favorable clinical outcomes for stage III-IVa NPC patients with good tolerance and similar toxicity compared to CCRT alone. A prospective, randomized clinical trial is essential to validate the current findings.

\section{Introduction}

Nasopharyngeal carcinoma (NPC) occurs commonly occurred in Southeast Asia and Southern China. It is characterized by a relatively high sensitivity to radiotherapy and chemotherapy (Y. Zhang et al., 2018). Radiotherapy with or without chemotherapy is the main treatment modality for NPC patients (Yan, Cao, \& Wang, 2017). According to the 2021 Chinese Society of Clinical Oncology and American Society of Clinical Oncology guidelines for nasopharyngeal carcinoma, concurrent chemoradiotherapy (CCRT) is still recommended as the standard treatment for stage II-IVa NPC patients (Yu-Pei Chen et al., 2021). Cisplatin-based chemotherapy with intensity-modulated radiotherapy (IMRT) is the standard care for NPC patients in recent years. Numerous studies have demonstrated that chemotherapy concurrent to radiation therapy provides better tumor control and more favorable outcomes (M. X. Zhang et al., 2015). However, nearly $30 \%$ of NPC patients may still experience treatment failure after CCRT, due to the relapse in situ and distant metastasis (Lee et al., 2005; Ma, Hui, \& Chan, 2008). The prognosis is poor for patients who diagnosed with distant metastasis, with the median survival time ranging from 5 to 11 months (Hui et al., 2004; Z. C. Zhang et al., 2014). Therefore, identification of a more effective therapeutic regimen with acceptable toxicity is urgently needed for NPC patients. 
The epidermal growth factor receptor (EGFR) is a member of the ErbB family of receptor tyrosine kinases. This receptor is over-expressed in about $90 \%$ of squamous cell carcinomas of the head and neck, including NPC (Fortunato Ciardiello \& Tortora, 2001; Yewale, Baradia, Vhora, Patil, \& Misra, 2013). In addition, the EGFR is a promising new therapeutic target in malignant carcinoma (Fortunato Ciardiello \& Tortora, 2001). Several EGFR inhibitors, such as cetuximab (CTX), panitumumab, afatinib, and erlotinib, have elicited positive outcomes in clinical trials (Dorsey \& Agulnik, 2013; Prenen, Vecchione, \& Van Cutsem, 2013; Yewale et al., 2013). Thus, anti-EGFR therapy may be a potential treatment combined with CCRT for NPC.

The most commonly used anti-EGFR monoclonal antibodies in the treatment of NPC are CTX and nimotuzumab (NTZ). Numerous previous studies have investigated the efficacy of anti-EGFR targeted therapy in NPC, though the results are controversial (Jian-feng Huang et al., 2017; Y. Li et al., 2017; Xia He et al., 2013; Xia et al., 2017; Xu et al., 2015; You, Hua, et al., 2017; You, Sun, et al., 2017). Xia et al. and You et al. found that the CTX or NTZ plus CCRT was more effective than CCRT alone (Xia et al., 2017; Xu et al., 2015). In contrast, $\mathrm{Li}$ et al. found that adding CTX to the CCRT regime did not improve the prognosis in stage II to IV NPC patients (Y. Li et al., 2017). Furthermore, the addition of CTX treatment significantly increased the toxicity rate of acute mucositis and acneiform rash (Y. Li et al., 2017; Xu et al., 2015). Compared with CTX, NTZ caused less severe dermatological toxicity, and exhibited a longer half-life (Tania Crombet et al., 2003). Previous studies with a small sample size have demonstrated that NTZ plus CCRT showed positive results for patients with locally advanced NPC (Zhi-gang Liu et al., 2016; Zhi-Qiang et al., 2019). Therefore, studies with a larger population are needed to validate the efficacy of adding NTZ.

Nomograms can assess the outcomes of individual patients by graphical depictions (Kattan \& Scardino, 2007), and they have been applied in various types of cancers (Han et al., 2012; Karakiewicz et al., 2007; Y. Wang et al., 2013). Furthermore, nomograms have been proposed as an alternative method to guide the therapeutic regimen for cancer patients, because of their specificity and visualization (Karakiewicz et al., 2007; Mariani et al., 2005). Thus, the primary aim of the present study was to evaluate the efficacy of NTZ in stage III-IVa NPC patients. A secondary aim was to develop a practical model by combining NTZ with prognostic biomarkers to predict the prognosis for NPC patients individually.

\section{Materials And Methods}

\section{Patient selection}

Between January 2015 and December 2017, a total of 11382 patients who were diagnosed with nasopharyngeal carcinoma at Sun Yat-sen University Cancer center were retrospectively enrolled. The pretreatment clinical information of the patients was recorded, including blood tests, imaging examination, and histological diagnosis. All patients had a histologic confirmation of NPC and enhanced computed tomography (CT)/MRI verified the metastatic disease and clinical stage. The exclusion criteria are presented in Fig. 1. Briefly, patients were ruled out if they had clinical stage I-II disease; distant metastasis; 
no/ unclear therapy; no receive concurrent chemotherapy; other targeted drugs; nimotuzumab less than 6 cycles; or lost to follow-up. Finally, 3340 patients were included in the study cohort, of them 3127 patients received CCRT alone, and 213 patients received CCRT combined with NTZ. In the 1:1 propensity score matching (PSM) analysis, patients who accepted nimotuzumab plus CCRT were individually matched to patients receiving CCRT alone according to body mass index (BMI), the levels of $C$ reactive protein (CRP) and lactate dehydrogenase (LDH), which were significantly different between the two treatment regimens on the patients' baseline characteristic. The current study was approved by the ethics committee of the SYSUCC (B2021-366-01). Written informed consent for therapy was obtained from all patients.

\section{Radiotherapy}

All patients received IMRT in Sun Yat-sen University Cancer Center. Briefly, gross tumor volume (GTVnx) included the primary tumor and the enlarged lymph nodes, and GTVnd was the volume of clinically positive cervical lymph nodes. The GTV, metastatic cervical lymph nodes and the metastatic retropharyngeal lymph nodes were verified by MRI/CT and/or PET-CT imaging as well as the nasopharyngoscopy findings. The first clinical tumor volume (CTV1) was defined as the gross tumor volume within $0.5-1.0 \mathrm{~cm}$ margin ( 0.2 to $0.3 \mathrm{~cm}$ posterior margin) to include the high-risk sites of microscopic extension and the nasopharynx. The second clinical tumor volume (CTV2) was derived as a geometric expansion of CTV1 plus 0.5-1.0 cm margin (0.2-0.3 cm posterior margin) to cover the low-risk areas of microscopic extension, the level of lymph node, and the potentially metastatic neck area. The radiotherapy dose in this retrospective study was ranged from 2.00 Gy to 2.33 Gy per fraction with five daily fractions per week for 6-7 weeks. The prescribed total radiation dose was 66-70 Gy to the planning target volume (PTV), 60 Gy to PTV1, 54 Gy to PTV2, and 60-66 Gy to the PTV of the involved cervical lymph nodes in 28 to 33 fractions. All patients received treatment once daily, five fractions weekly. Dose were applied within the tolerance according to the RTOG 0225 protocol.

\section{EGFR-antibody therapy and chemotherapy}

All patients received cisplatin-based concurrent chemotherapy, and the treatment regimen of concurrent chemotherapy was $100 \mathrm{mg} / \mathrm{m}^{2}$ cisplatin on day 1 every 3 weeks, or $30 \mathrm{mg} / \mathrm{m}^{2}$ cisplatin weekly. Induction chemotherapy (IC) consisted of cisplatin combined with taxanes or 5-fluorouracil or both every 3 weeks for 2 or 3 cycles (Y. Sun et al., 2016). A total of 213 patients accepted full doses of nimotuzumab therapy (200 mg weekly) after PSM in our study.

\section{Toxicity assessment and follow up}

Chemotherapy toxicities were evaluated by the National Cancer Institute Common Toxicity Criteria (NCl CTCAE, version 4.0), and radiotherapy-related toxicities were assessed according to the Acute and Late Radiation Morbidity Scoring Criteria of RTOG. Dosage was adjusted based on the grade of toxicities individually. Patients were monitored weekly during treatment. The post-treatment clinical follow-up was generally at intervals of 3 months after treatment for first 2 years, half-year for the next 3 years and annually thereafter. Assessments included patient history, physical and imaging examination of the nasopharynx. The last follow-up date was 31 December 2020. 


\section{Study end points and statistical analysis}

Overall survival (OS) was defined as the time from the date of enrollment to the date of death due to any cause or the date of last follow-up. Progression-free survival (PFS), locoregional recurrence-free survival (LRFS), and distant metastasis-free survival (DMFS) were calculated from day after the completion of therapy to the first diagnosed of any cause of treatment failure, locoregional nasopharynx/ cervical lymph nodes relapse, and the distant recurrence/ metastasis, respectively, or the date of last investigation.

The baseline characteristics of patients are described in the CCRT plus NTZ group and CCRT alone group. The $\chi 2$ test was used to compared categorical variables and the $t$-test was applied to compared continuous variables. Propensity score matching analysis was applied. Using a caliper width of 0.2, 1:1 matching was performed in two groups based on the propensity scores. OS, PFS, LRFS, and DMFS were calculated using the Kaplan-Meier method. Cox analysis was performed to identify the significant prognostic factors. Variables as independently prognostic factors in the multivariable models were included to construct a nomogram model. Calibration curves of the nomogram were plotted to compare predicted survival with actual survival. The statistical analyses were executed by the SPSS 20.0 (Chicago, IL, USA) and using R (version 3.2.3). All tests were two-sided, and $P<0.05$ was considered statistically significant.

\section{Results}

\section{Patient Characteristics and Follow-up}

From January 2015 to December 2017, 3340 NPC carcinoma patients were enrolled in our retrospective study. Patient characteristics, grouped by the treatment regimen (with or without NTZ), are detailed in Supplemental Table 1. The distributions of BMI, and the serum levels of CRP and LDH, were significantly different between the two groups $(P<0.05)$ (Supplemental Table 1). To eliminate potential confounding factors, we included a well-balanced cohort via PSM. After PSM, 426 eligible patients were identified for the matched analysis. No significant difference was observed in the clinical characteristics between the two regimens in the PSM cohort (Table 1). For the PSM cohorts, the median follow-up times were 60.0 months (range from 3 to 60 months). The follow-up observation indicated 17 and 4 patients died in the PSM CCRT and CCRT plus NTZ cohorts, respectively. 
Table 1

Comparison of Patient Characteristics After Propensity Score Matching Grouped by Treatment Regime.

\begin{tabular}{|c|c|c|c|}
\hline Characteristic & CCRT $(n=213)$ & CCRT+NTZ $(n=213)$ & $P$ \\
\hline Gender & & & 0.661 \\
\hline Female & $59(27.7)$ & $54(25.4)$ & \\
\hline Male & 154(72.3) & $159(74.6)$ & \\
\hline Age & & & 0.332 \\
\hline$<45 y$ & $118(55.4)$ & $107(50.2)$ & \\
\hline$\geq 45 y$ & $95(44.6)$ & $106(49.8)$ & \\
\hline BMI & & & 1.000 \\
\hline$<25$ & $141(66.2)$ & $141(66.2)$ & \\
\hline$\geq 25$ & $72(33.8)$ & $72(33.8)$ & \\
\hline T stage & & & 0.874 \\
\hline $\mathrm{T} 1$ & $21(9.9)$ & $17(8.0)$ & \\
\hline T2 & $9(4.2)$ & $8(3.8)$ & \\
\hline T3 & $137(64.3)$ & $144(67.6)$ & \\
\hline $\mathrm{T} 4$ & $46(21.6)$ & $44(20.7)$ & \\
\hline N stage & & & 0.844 \\
\hline NO & $25(11.7)$ & $30(14.1)$ & \\
\hline N1 & $93(43.7)$ & $86(40.4)$ & \\
\hline N2 & $53(24.9)$ & $56(26.3)$ & \\
\hline N3 & $42(19.7)$ & $41(19.2)$ & \\
\hline Clinical stage & & & 1.000 \\
\hline III & $132(62.0)$ & $133(62.4)$ & \\
\hline $\mathrm{IVa}$ & $81(38.0)$ & $80(37.6)$ & \\
\hline EBV DNA (copies/ml) & & & 0.438 \\
\hline$<2500$ & $102(47.9)$ & $111(52.1)$ & \\
\hline
\end{tabular}

Data are mean $+\mathrm{SD}$ or $\mathrm{n}(\%)$.

Abbreviations: CCRT, concurrent chemoradiotherapy; NTZ, nimotuzumab; BMI, body mass index; EBV, Epstein-Barr virus; LDH, lactate dehydrogenase; CRP, $\mathrm{C}$ reactive protein. 


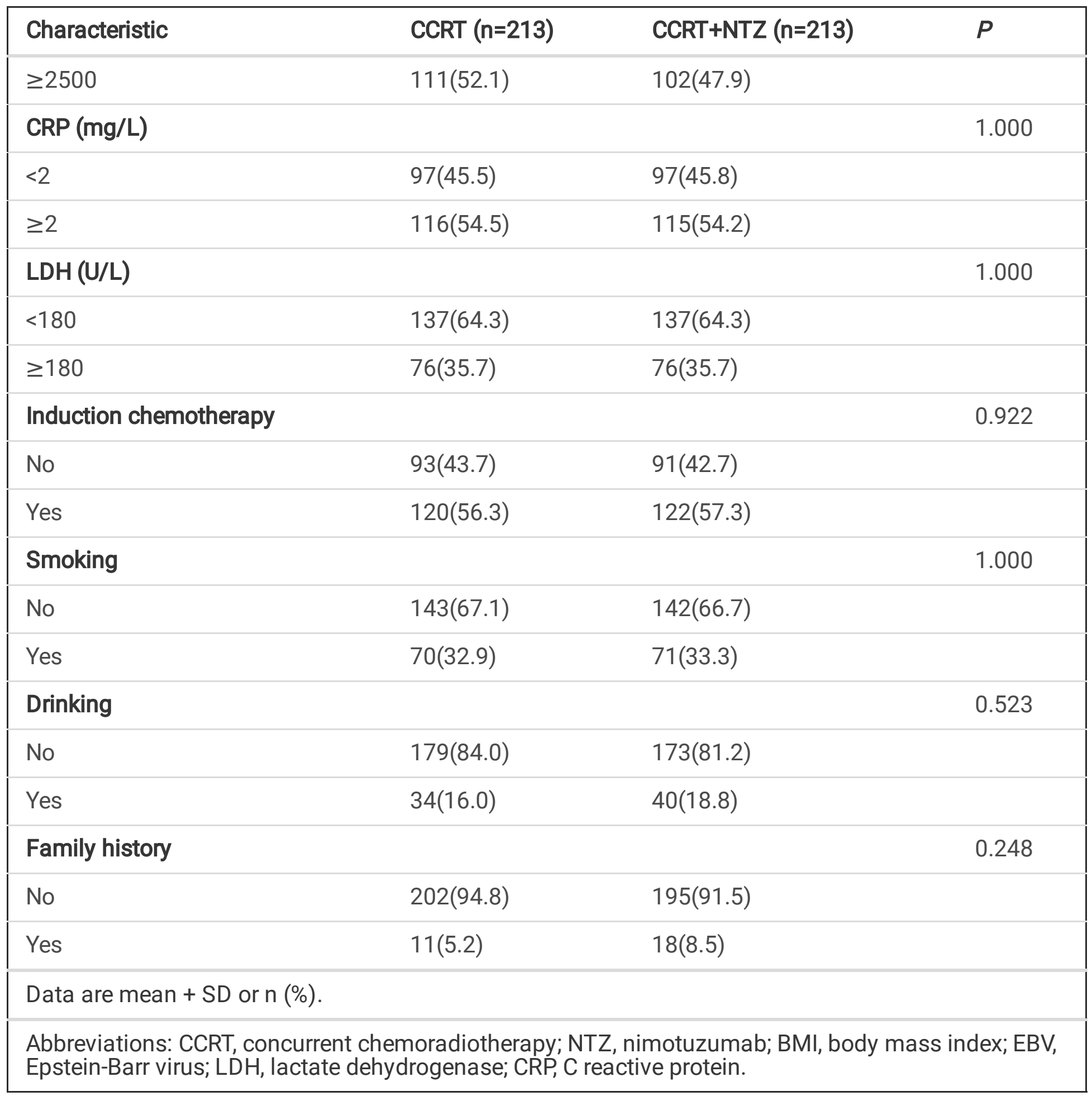

\section{Efficacy and Safety of NTZ}

We further investigated whether NPC patients could benefit from the regimen of NTZ after CCRT in the PSM cohort. The 1-, 3-, and 5-year OS were $90.1 \%, 87.8 \%, 72.3 \%$ and $96.7 \%, 92.0 \%, 76.1 \%$, the 1-, 3-, and 5year PFS were $90.6 \%, 85.9 \%, 69.0 \%$ and $97.2 \%, 95.3 \%, 73.2 \%$, the $1-, 3-$, and 5 -year LRFS were $92.5 \%$, $86.4 \%, 69.0 \%$ and $97.7 \%, 92.5 \%, 73.2 \%$, and the $1-, 3$-, and 5 -year DMFS were $94.3 \%, 87.3 \%, 69.0 \%$ and $98.1 \%, 93.0 \%, 73.2 \%$ for the CCRT group and NTZ plus CCRT group, respectively. 
The Kaplan-Meier curves presented in Fig. 2 show that patients in the NTZ plus CCRT group had significantly better overall survival (log rank=8.394, $P=0.004$ ), PFS (log rank=9.486, $P=0.002$ ), and LRFS (log rank=4.810, $P=0.028$ ) than NPC patients given CCRT alone (Fig. 2a-C). In addition, patients who received the NTZ plus CCRT treatment had favorable DMFS than those who received CCRT alone, at the very edge of significance (log rank=3.741, $P=0.053)$ (Fig. 2d).

We performed a Cox regression analysis to identify potential prognostic factors for NPC patients. All 426 patients after PSM were included in the Cox analysis. According to the univariate analysis, NTZ plus CCRT, age, clinical stage, and the levels of EBV DNA, and LDH before treatment were associated with OS (Fig. 3a). Similarly, NTZ combined with CCRT, clinical stage, and the level of EBV DNA were associated with PFS (Fig. 3b). For LRFS, NTZ plus CCRT was suggested as a potential prognostic factor (Fig. 3c). However, no clinicopathological feature was found to be related to the DMFS (Fig. 3d).

Consequently, we conducted a multivariate analysis adjusted for confounding factors. As presented in Fig. 4, the combination of NTZ and CCRT (hazard ratio (HR) $=0.192 ; 95 \%$ confidence interval $(\mathrm{Cl})$ : $0.064-$ $0.576, P=0.003$ ) was identified as an independent protective factors for OS, while age ( $\geq 45$ years), clinical stage, and the levels of $\mathrm{LDH}(\geq 180 \mathrm{U} / \mathrm{L})$ were shown to be independent adverse prognostic factors (Fig. 4a). Meanwhile, the regimen of NTZ after CCRT was demonstrated as an independent protective factors for PFS (HR $=0.171 ; 95 \% \mathrm{Cl}: 0.050-0.592, P=0.005)$ and LRFS (HR $=0.206 ; 95 \% \mathrm{Cl}$ : 0.044-0.957, $P=0.044$ ), but not for DMFS (HR =0.138; 95\% Cl: 0.061-1.196, $P=0.072)$ (Fig. 4b-d). Interestingly, induction therapy was not associated with DMFS in the univariate model, but it showed a significant protective effect for DMFS in the multivariate analysis (HR $=0.148 ; 95 \% \mathrm{Cl}: 0.025-0.891, P$ $=0.037)$ (Fig. 4d).

The incidence of acute toxicity in the PSM cohort was evaluated between CCRT with and without NTZ. There were no significant differences among the two regimens in major toxicities such as anemia, thrombocytopenia, leukocytopenia, skin eruption, mucositis and organ toxicity (all $P>0.05$ ) (Table 2 ). In addition, we evaluated the interaction effects between the two different treatment regimens and other prognostic factors after adjusting for age, clinical stage, induction chemotherapy, and EBV DNA. There were no significant interaction effects found between the therapeutic regimens and other prognostic factors on OS (all $P>0.05$ ) (Supplemental Table 2). 
Table 2

Acute toxicities based on different treatment regimen in NPC patients of well-balanced cohort.

\begin{tabular}{|llll|}
\hline Acute Toxicity & CCRT & CCRT plus NTZ & $P$ \\
\hline Anemia & & & 0.679 \\
\hline G0-G1 & $170(79.7)$ & $176(82.6)$ & \\
\hline G2 & $34(16.0)$ & $30(14.1)$ & \\
\hline G4 & $7(3.5)$ & $4(2.2)$ & \\
\hline Thrombocytopenia & $2(0.9)$ & $2(1.1)$ & 0.177 \\
\hline G0-G1 & & & \\
\hline G2 & $183(85.5)$ & $193(90.4)$ & \\
\hline G3 & $23(11.0)$ & $17(8.1)$ & \\
\hline G4 & $5(2.3)$ & $2(0.9)$ & \\
\hline Neutropenia & $2(1.2)$ & $1(0.6)$ & \\
\hline G0-G1 & & & \\
\hline G2 & $144(67.4)$ & $160(75.3)$ & \\
\hline G3 & $14(6.7)$ & $10(4.9)$ & \\
\hline G4 & & & \\
\hline Leukopenia & $34(20.3)$ & $34(15.7)$ & \\
\hline G0-G1 & $23(11.0)$ & $18(8.4)$ & \\
\hline G2 & $2(1.2)$ & $1(0.6)$ & \\
\hline G3 & & & \\
\hline G4 & $36(17.2)$ & $32(15.1)$ & \\
\hline Skin reaction & & $2(0.9)$ & \\
\hline G0-G1 & & & \\
\hline G2 & & & \\
\hline G3 & & & \\
\hline Mucositis & & & \\
\hline
\end{tabular}

Abbreviations: CCRT, concurrent chemoradiotherapy; NTZ, nimotuzumab. 


\begin{tabular}{|llll|}
\hline Acute Toxicity & CCRT & CCRT plus NTZ & $P$ \\
\hline G0-G1 & $57(26.6)$ & $70(32.8)$ & \\
\hline G2 & $86(40.5)$ & $77(36.3)$ & \\
\hline G3 & $66(30.9)$ & $56(26.2)$ & 0.373 \\
\hline Nausea and vomiting & $4(2)$ & $10(4.7)$ & \\
\hline G0-G1 & & & \\
\hline G2 & $64(29.9)$ & $72(33.7)$ & \\
\hline G3 & $116(54.7)$ & $106(50.0)$ & \\
\hline G4 & $30(14.0)$ & $34(15.7)$ & \\
\hline Diarrhea & $3(1.5)$ & $1(0.3)$ & \\
\hline G0-G1 & & & \\
\hline G2 & $189(88.7)$ & $182(85.5)$ & \\
\hline G3 & $19(9)$ & $24(11.3)$ & \\
\hline Hepatoxicity & $5(2.3)$ & $7(3.2)$ & \\
\hline G0-G1 & & & \\
\hline G2 & $152(71.2)$ & $158(74.4)$ & \\
\hline G3 & $194(28.8)$ & $55(25.6)$ & \\
\hline Nephrotoxicity & $189(88.7)$ & $180(84.6)$ & \\
\hline G0-G1 & $19(9.0)$ & $25(11.9)$ & \\
\hline G2 & $5(2.3)$ & $7(3.5)$ & \\
\hline G3 & & & \\
\hline Weight loss & & & \\
\hline G0-G1 & & & \\
\hline G2 & & & \\
\hline Abbreviations: CCRT, concurrent chemoradiotherapy; NTZ, nimotuzumab. & \\
\hline
\end{tabular}


Potential prognostic factors were utilized to construct a nomogram and predict the prognosis of each patient specifically. Prognostic models were developed for OS, PFS, LRFS, and DMFS, respectively (Fig. 5). The four models illustrated good accuracy for predicting the survival rate of NPC patients. When the nomogram models were authenticated internally, with the 1000 bootstrap resampling technique, the concordance indexes (c-index) of 0.867 (95\% Cl, 0.793-0.941), 0.822 (95\% Cl, 0.743-0.901), 0.816 (95\% Cl, 0.702-0.930), and 0.848 (95\% Cl, 0.708-0.988) for OS, PFS, LRFS, and DMFS were attained, respectively. The calibration plots demonstrated a favorable relation between the predicted and observed survival (Fig. 5). Smaller points in the nomogram indicated a better prognosis. The models indicated that NTZ plus CCRT had a positive impact on the survival NPC patients.

\section{Discussion}

The current study evaluated the therapeutic effect of nimotuzumab combined with concurrent chemoradiotherapy in stage III-IVa nasopharyngeal carcinoma patients, and demonstrated that this combination achieved better prognosis compared with CCRT alone, with similar toxicity rates between the two regimens. Furthermore, we constructed nomogram models to assess the survival probability according to the clinical characteristics of individual patients.

Although nasopharyngeal tumors are sensitive to radiotherapy, some patients still develop recurrence and metastases after treatment. EGFR is over-expressed in most NPC patients, and its high expression level is an indication of strong invasion and drug resistance (Fortunato Ciardiello \& Tortora, 2001). Several reports have examined the efficacy of NTZ treatment for NPC patients, but the conclusions are discrepant (Fangzheng et al., 2018; Jian-feng Huang et al., 2017; F. Wang et al., 2018; Yao et al., 2018; Zhi-gang Liu et al., 2016; Zhi-Qiang et al., 2019). For example, Li et al. conducted a retrospective study and found that NPC patients receiving cisplatin and radiotherapy achieved better survival than those receiving NTZ combined with radiotherapy, while NTZ plus radiotherapy caused less toxicity. They suggested NTZ plus radiotherapy is an optional treatment for the stage II or older NPC patients (H. M. Li et al., 2016). However, NPC patients in stage II rarely receive anti-EGFR drugs treatment in our cancer center. Therefore, only stage III-IVa NPC patients are discussed in the current study, and the therapeutic effect of the NTZ combination for stage II NPC patients remains elusive. Liu et al. conducted a study retrospectively and concluded that NTZ combined with CCRT was an effective treatment option for locoregionally advanced NPC (Zhi-gang Liu et al., 2016). Similarly, Yao et al. suggested that NTZ in addition to the standard CCRT regimen was more effective for long-term survival in locoregionally advanced NPC patients in comparison to CCRT alone. Nevertheless, only 42 and 31 patients treated with CCRT plus NTZ were included in the two previous studies, and the results are thus inconclusive (Yao et al., 2018; Zhi-gang Liu et al., 2016).

Our cancer center previously documented the efficacy of combination anti-EGFR targeted treatment. You et al. first reported that significantly longer survival rates were not observed in patients receiving CTX/NTZ treatment concurrently with IMRT compared to those in the standard cisplatin-IMRT combination. Furthermore, the subgroup analysis revealed frustrating loco-regional control in N3 stage 
patients treated with CTX/NTZ. Additionally, G3-G4 hematologic toxicities and an increased rate of CTXrelated mucositis were observed in the CTX/NTZ group (You, Sun, et al., 2017). You et al. then compared the efficacy of CTX or NTZ and CCRT, and CCRT treatment alone, in stage II-IVa NPC patients. They suggested that the combination of CTX/NTZ with CCRT yielded better OS in patients with stage II-IVa NPC compared with CCRT alone. In addition, the positive effect of the combined CTX/NTZ treatment was also evident in DFS and DMFS (You, Hua, et al., 2017).

Our data are partially consistent with these previous findings. In the current study, the combination of NTZ and CCRT improved OS, PFS, and LRFS for stage III-IVa NPC patients without increasing the incidence of acute toxicity. However, DMFS did not benefit from the addition of NTZ treatment (Fig. 2 and Fig. 3). We hypothesize that the inconsistent results might be due to a discrepancy in patient enrollment. Specifically, the analysis by You et al. included patients between January 2009 and December 2013, with 189 patients in the CTX/NTZ plus CCRT arm. In addition, the molecularly-targeted drug was CTX or NTZ, not specifically NTZ, and eligible patients received only one treatment cycle. In the current study, we enrolled NPC patients from 2015 to 2017, and focused on the efficacy of NTZ specifically. Patients receiving at least six doses of NTZ were included, and 213 eligible patients were assigned to each cohort using the PSM method. In addition, Sun et al. conducted a study to investigate the efficacy of anti-EGFR drugs added to palliative chemotherapy in patients with de novo metastatic NPC. They reported that de novo metastatic NPC patients might not benefit from the addition of anti-EGFR drug treatment, and adding CTX to CCRT might exacerbate the acute mucositis and skin reactions (X.-S. Sun et al., 2019). Collectively, the efficacy of anti-EGFR drugs on suppressing distant metastasis remains to be determined.

NTZ is a recombinant humanized IgG1 monoclonal antibody that binds to EGFR directly (Cristina Mateo et al., 1997). Therefore, NTZ can inhibit the binding of EGF and transforming growth factor alpha to the EGFR, and thereby block the EGFR signaling pathway. In addition, NTZ is reported to induce apoptosis, and inhibit the cell cycle and angiogenesis in tumors (Crombet-Ramos, Rak, Perez, \& Viloria-Petit, 2002). Furthermore, NTZ has high safety and low toxicity, with a relatively low risk of skin and mucosa toxicities commonly caused by other EGFR-targeting antibodies (Lin et al., 2018; Zhi-Qiang et al., 2019). These factors suggest that NTZ has advantages over other EGFR inhibitors, such as CTX, in terms of less toxicity (Lin et al., 2018). In addition, the affinity constant of NTZ is low, which leads to highly specific tumor uptake and low normal tissue absorption.

Previous clinical trials that studied the efficacy of NTZ combined with radiotherapy in patients with locally advanced head and neck squamous cell carcinoma reported that the addition of NTZ caused mild toxicity and may improve the radiation sensitivity of unresectable head and neck tumors (Talavera et al., 2009). Randomized studies that enrolled Indian patients with advanced head and neck squamous cell carcinoma concluded that concurrent use of NTZ with radiotherapy or chemoradiotherapy was safe and provided long-term survival benefits (Patil et al., 2019; Reddy et al., 2014). In NPC, CCRT significantly increased radiotherapy- and chemotherapy-related adverse events such as mucositis, skin reactions, and organ toxicity (Chen et al., 2012). Therefore, it is essential to clarify the adverse side effects of combination treatments with NTZ. Wang et al. concluded that long-term usage of NTZ did not increase 
the incidence of radiation-related toxicities; no skin rashes or infusion reactions were observed in the treated NPC patients (Fangzheng et al., 2018). Consistent with this finding, Wang et al. conducted a 1:3 PSM analysis in III-IVa stage NPC patients that received IMRT with or without NTZ, and no significant differences were observed between the two regimens in terms of acute toxicities (Zhi-Qiang et al., 2019). We made similar observations in the current analysis (Table 2), demonstrating that NTZ plus CCRT may be a safe therapy regimen for locally advanced NPC patients.

The nomogram models showed favorable predictive accuracy and discriminative ability. Some studies have established a nomogram model for predicting NPC survival (J. Li et al., 2018; W.-Z. Li et al., 2021; Tang et al., 2015). In the present study, we constructed models for predicting the OS, PFS, LRFS, and DMFS. We included potential prognostic biomarkers in the nomogram models. The models showed that the combination of NTZ and CCRT treatment contributed to better predict prognosis for stage III-IVa NPC patients.

Although our analysis verified the positive efficacy of the NTZ plus CCRT regimen for local advanced NPC patients, and the nomogram models provided useful tools for clinical decision-making, the current study still had several limitations. Firstly, as a retrospective study, potential selection bias was unavoidable, even though the bias was minimized by recruiting consecutive NPC patients and using the PSM analysis. Secondly, information of the EGFR expression level is missing; therefore, the relationship between the EGFR expression level and the efficacy of NTZ is unclear. Finally, the study population was enrolled from a single center. Therefore, external validation, including a prospective and randomized study, is needed to verify our findings.

\section{Conclusion}

The present study suggested that the combination of NTZ with CCRT resulted in a favorable clinical OS outcome for stage III-IVa NPC patients with good tolerance and similar toxicities compared to CCRT alone. In addition, we established useful nomogram models to evaluate the benefit of CCRT in combination with NTZ for individual NPC patients, which could help in making individual treatment decisions.

\section{Abbreviations}




\begin{tabular}{|c|c|}
\hline CCRT & Concurrent chemoradiotherapy \\
\hline NTZ & Nimotuzumab \\
\hline NPC & Nasopharyngeal carcinoma \\
\hline OS & Overall survival \\
\hline PFS & Progression-free survival \\
\hline LRFS & Locoregional recurrence-free survival \\
\hline DMFS & Distant metastasis-free survival \\
\hline PSM & Propensity score matching \\
\hline $\operatorname{csco}$ & Chinese Society of Clinical Oncology \\
\hline IMRT & Intensity-modulated radiotherapy \\
\hline EGFR & Epidermal growth factor receptor \\
\hline CTX & Cetuximab \\
\hline CT & Computed tomography \\
\hline GTV & Gross tumor volume \\
\hline CTV & Clinical tumor volume \\
\hline BMI & Body mass index \\
\hline LDH & Lactate dehydrogenase \\
\hline CRP & $\mathrm{C}$ reactive protein \\
\hline C-index & Concordance index \\
\hline PFS & Progression free survival \\
\hline IC & Induction chemotherapy \\
\hline
\end{tabular}

\section{Declarations}

\section{Funding}

This work was supported by grants from the National Natural Science Foundation of China (grant numbers 81872375 and 82172863 ) and the Natural Science Foundation of Guangdong Province (grant numbers 2021A1515010118).

\section{Competing interest}

Conflict of interest relevant to this article was not reported. 


\section{Author contributions}

Zhuochen Cai developed the study concepts and design. Chixiong Liang and Yingying Huang participated in the data acquisition. Dongni Chen and Wenze Qiu participated in the data analysis and interpretation. Zhuochen Cai, Jiayu Zhou and Zejiang Zhan participated in the manuscript writing and editing. Yanqun Xiang, Xiang Guo and Xing Lv participated in the manuscript review. All authors contributed to the article and approved the submitted version.

\section{Data availability}

The datasets for the current study are available on request to the corresponding authors.

\section{Ethics approval}

This retrospective study was approved by the Clinical Research Committee of Sun Yat-sen University Cancer Center (B2021-366-01).

\section{Consent to participate}

Written informed consent was obtained from each patient for the publication.

\section{Consent to publish}

Not applicable.

\section{Acknowledgements}

We are grateful to our patients and staffs who involved in the patient care to make this work possible. We kindly thank the editor and reviewers for their careful review and valuable comments, which have helped significantly improve the manuscript.

\section{References}

1. Chen, L., Hu, C.-S., Chen, X.-Z., Hu, G.-Q., Cheng, Z.-B., Sun, Y., . . Ma, J. (2012). Concurrent chemoradiotherapy plus adjuvant chemotherapy versus concurrent chemoradiotherapy alone in patients with locoregionally advanced nasopharyngeal carcinoma: a phase 3 multicentre randomised controlled trial. The Lancet Oncology, 13(2), 163-171. doi:10.1016/s14702045(11)70320-5

2. Cristina Mateo, Ernest0 Moreno, Kathryn Amour, Josefa Lombardero, William Harris, \& Perez, R. (1997). Humanization of a mouse monoclonal antibody that blocks the epidermal growth factor receptor: recovery of antagonistic activity. Immunotechnology, 3, 71-81.

3. Crombet-Ramos, T., Rak, J., Perez, R., \& Viloria-Petit, A. (2002). Antiproliferative, antiangiogenic and proapoptotic activity of h-R3: A humanized anti-EGFR antibody. Int J Cancer, 101(6), 567-575. 
doi:10.1002/ijc.10647

4. Dorsey, K., \& Agulnik, M. (2013). Promising new molecular targeted therapies in head and neck cancer. Drugs, 73(4), 315-325. doi:10.1007/s40265-013-0025-3

5. Fangzheng, W., Chuner, J., Zhiming, Y., Tongxin, L., Fengqin, Y., Lei, W., . . Zhenfu, F. (2018). LongTerm Use of Nimotuzumab in Combination With Intensity-Modulated Radiotherapy and Chemotherapy in the Treatment of Locoregionally Advanced Nasopharyngeal Carcinoma: Experience of a Single Institution. Oncology Research Featuring Preclinical and Clinical Cancer Therapeutics, 26(2), 277-287. doi:10.3727/096504017x15079846743590

6. Fortunato Ciardiello, \& Tortora, G. (2001). A Novel Approach in the Treatment of Cancer: Targeting the Epidermal Growth Factor Receptor. Clinical Cancer Research, 7, 2958-2970.

7. Han, D. S., Suh, Y. S., Kong, S. H., Lee, H. J., Choi, Y., Aikou, S., . . Yang, H. K. (2012). Nomogram predicting long-term survival after d2 gastrectomy for gastric cancer. J Clin Oncol, 30(31), 38343840. doi:10.1200/JC0.2012.41.8343

8. Hui, E. P., Leung, S. F., Au, J. S., Zee, B., Tung, S., Chua, D., . . Chan, A. T. (2004). Lung metastasis alone in nasopharyngeal carcinoma: a relatively favorable prognostic group. A study by the Hong Kong Nasopharyngeal Carcinoma Study Group. Cancer, 101(2), 300-306. doi:10.1002/cncr.20358

9. Jian-feng Huang, Fu-zheng Zhang, C-M Charlie Ma, Qin-zhou Zou, Le-yuan Zhou, Jian-jun Chu, ... Ma, C.-M. C. (2017). Induction chemotherapy followed by concurrent chemoradiation and nimotuzumab for locoregionally advanced nasopharyngeal carcinoma: preliminary results from a phase II clinical trial. Oncotarget, 8, 2754-2465.

10. Karakiewicz, P. I., Briganti, A., Chun, F. K., Trinh, Q. D., Perrotte, P., Ficarra, V., . . Patard, J. J. (2007). Multi-institutional validation of a new renal cancer-specific survival nomogram. J Clin Oncol, 25(11), 1316-1322. doi:10.1200/JCO.2006.06.1218

11. Kattan, M. W., \& Scardino, P. T. (2007). Evidence for the usefulness of nomograms. Nat Clin Pract Urol, 4(12), 638-639. doi:10.1038/ncpuro0968

12. Lee, A. W., Sze, W. M., Au, J. S., Leung, S. F., Leung, T. W., Chua, D. T., . . Lau, W. H. (2005). Treatment results for nasopharyngeal carcinoma in the modern era: the Hong Kong experience. Int J Radiat Oncol Biol Phys, 61(4), 1107-1116. doi:10.1016/j.ijrobp.2004.07.702

13. Li, H. M., Li, P., Qian, Y. J., Wu, X., Xie, L., Wang, F., . . Liu, L. (2016). A retrospective paired study: efficacy and toxicity of nimotuzumab versus cisplatin concurrent with radiotherapy in nasopharyngeal carcinoma. BMC Cancer, 16(1). doi:10.1186/s12885-016-2974-x

14. Li, J., Chen, S., Peng, S., Liu, Y., Xing, S., He, X., \& Chen, H. (2018). Prognostic nomogram for patients with Nasopharyngeal Carcinoma incorporating hematological biomarkers and clinical characteristics. International Journal of Biological Sciences, 14(5), 549-556. doi:10.7150/ijbs.24374

15. Li, W.-Z., Lv, S.-H., Liu, G.-Y., Liang, H., Guo, X., Lv, X., . . Xiang, Y.-Q. (2021). Development of a Prognostic Model to Identify the Suitable Definitive Radiation Therapy Candidates in de Novo Metastatic Nasopharyngeal Carcinoma: A Real-World Study. International Journal of Radiation Oncology ${ }^{*}$ Biology ${ }^{*}$ hysics, 109(1), 120-130. doi:10.1016/j.ijrobp.2020.08.045 
16. Li, Y., Chen, Q.-Y., Tang, L.-Q., Liu, L.-T., Guo, S.-S., Guo, L., . . Mai, H.-Q. (2017). Concurrent chemoradiotherapy with or without cetuximab for stage II to IVb nasopharyngeal carcinoma: a casecontrol study. BMC Cancer, 17(1). doi:10.1186/s12885-017-3552-6

17. Lin, M., You, R., Liu, Y.-P., Zhang, Y.-N., Zhang, H.-J., Zou, X., . . Chen, M.-Y. (2018). Beneficial effects of anti-EGFR agents, Cetuximab or Nimotuzumab, in combination with concurrent chemoradiotherapy in advanced nasopharyngeal carcinoma. Oral Oncology, 80, 1-8. doi:10.1016/j.oraloncology.2018.03.002

18. Ma, B. B., Hui, E. P., \& Chan, A. T. (2008). Systemic approach to improving treatment outcome in nasopharyngeal carcinoma: current and future directions. Cancer Sci, 99(7), 1311-1318. doi:10.1111/j.1349-7006.2008.00836.x

19. Mariani, L., Miceli, R., Kattan, M. W., Brennan, M. F., Colecchia, M., Fiore, M., .. Gronchi, A. (2005). Validation and adaptation of a nomogram for predicting the survival of patients with extremity soft tissue sarcoma using a three-grade system. Cancer, 103(2), 402-408. doi:10.1002/cncr.20778

20. Patil, V. M., Noronha, V., Joshi, A., Agarwal, J., Ghosh-Laskar, S., Budrukkar, A., . . Prabhash, K. (2019). A randomized phase 3 trial comparing nimotuzumab plus cisplatin chemoradiotherapy versus cisplatin chemoradiotherapy alone in locally advanced head and neck cancer. Cancer, 125(18), 3184-3197. doi:10.1002/cncr.32179

21. Prenen, H., Vecchione, L., \& Van Cutsem, E. (2013). Role of targeted agents in metastatic colorectal cancer. Target Oncol, 8(2), 83-96. doi:10.1007/s11523-013-0281-x

22. Reddy, B. K. M., Lokesh, V., Vidyasagar, M. S., Shenoy, K., Babu, K. G., Shenoy, A., . . Tanvir Pasha, C. R. (2014). Nimotuzumab provides survival benefit to patients with inoperable advanced squamous cell carcinoma of the head and neck: A randomized, open-label, phase Ilb, 5-year study in Indian patients. Oral Oncology, 50(5), 498-505. doi:10.1016/j.oraloncology.2013.11.008

23. Sun, X.-S., Liang, Y.-J., Li, X.-Y., Liu, S.-L., Chen, Q.-Y., Tang, L.-Q., \& Mai, H.-Q. (2019). Palliative chemotherapy with or without anti-EGFR therapy for de novo metastatic nasopharyngeal carcinoma: a propensity score-matching study. Drug Design, Development and Therapy, Volume 13, 3207-3216. doi:10.2147/dddt.S215190

24. Sun, Y., Li, W.-F., Chen, N.-Y., Zhang, N., Hu, G.-Q., Xie, F.-Y., . . Ma, J. (2016). Induction chemotherapy plus concurrent chemoradiotherapy versus concurrent chemoradiotherapy alone in locoregionally advanced nasopharyngeal carcinoma: a phase 3, multicentre, randomised controlled trial. The Lancet Oncology, 17(11), 1509-1520. doi:10.1016/s1470-2045(16)30410-7

25. Talavera, A., Friemann, R., Gomez-Puerta, S., Martinez-Fleites, C., Garrido, G., Rabasa, A., . . Moreno, E. (2009). Nimotuzumab, an antitumor antibody that targets the epidermal growth factor receptor, blocks ligand binding while permitting the active receptor conformation. Cancer Res, 69(14), 58515859. doi:10.1158/0008-5472.CAN-08-4518

26. Tang, L.-Q., Li, C.-F., Li, J., Chen, W.-H., Chen, Q.-Y., Yuan, L.-X., . . Z Zeng, M.-S. (2015). Establishment and Validation of Prognostic Nomograms for Endemic Nasopharyngeal Carcinoma. Journal of the National Cancer Institute, 108(1). doi:10.1093/jnci/djv291 
27. Tania Crombet, Leonel Torres, Elia Neninger, Mauricio Catala, Marı́a E. Solano, Alejandro Perera, ... Lage, A. n. (2003). Pharmacological Evaluation of Humanized Anti-Epidermal Growth Factor Receptor, Monoclonal Antibody h-R3, in Patients With Advanced Epithelial-Derived Cancer. Journal of Immunotherapy, 26, 139-148.

28. Wang, F., Sun, Q., Jiang, C., Liu, T., Rihito, A., Masoto, S., ... Chen, M. (2018). Additional induction chemotherapy to concurrent chemotherapy and intensity-modulated radiotherapy with or without nimotuzumab in first-line treatment for locoregionally advanced nasopharyngeal carcinoma: a propensity score matched analysis. J Cancer, 9(3), 594-603. doi:10.7150/jca.20461

29. Wang, Y., Li, J., Xia, Y., Gong, R., Wang, K., Yan, Z., . . Shen, F. (2013). Prognostic nomogram for intrahepatic cholangiocarcinoma after partial hepatectomy. J Clin Oncol, 31(9), 1188-1195. doi:10.1200/JCO.2012.41.5984

30. Xia He, Jianhua Xu, Wenjie Guo, Xuesong Jiang, Xue Wang, \& Zong, D. (2013). Cetuximab in combination with chemoradiation after induction chemotherapy of locoregionally advanced nasopharyngeal carcinoma: preliminary results. Future Oncol., 9, 1459-1467.

31. Xia, W.-X., Liang, H., Lv, X., Wang, L., Qian, C.-N., Ye, Y.-F., . . Guo, X. (2017). Combining cetuximab with chemoradiotherapy in patients with locally advanced nasopharyngeal carcinoma: A propensity score analysis. Oral Oncology, 67, 167-174. doi:10.1016/j.oraloncology.2017.02.026

32. Xu, T., Liu, Y., Dou, S., Li, F., Guan, X., \& Zhu, G. (2015). Weekly cetuximab concurrent with IMRT aggravated radiation-induced oral mucositis in locally advanced nasopharyngeal carcinoma: Results of a randomized phase II study. Oral Oncology, 51(9), 875-879.

doi:10.1016/j.oraloncology.2015.06.008

33. Yan, H., Cao, X., \& Wang, J. (2017). Application of intensity-modulated radiation therapy in the treatment of nasopharyngeal carcinoma. Oncol Lett, 14(6), 7773-7776. doi:10.3892/ol.2017.7186

34. Yao, J. J., Zhang, L. L., Gao, T. S., Peng, Y. L., Lawrence, W. R., Zhang, W. J., . . Sun, Y. (2018). Comparing treatment outcomes of concurrent chemoradiotherapy with or without nimotuzumab in patients with locoregionally advanced nasopharyngeal carcinoma. Cancer Biol Ther, 19(12), 11021107. doi:10.1080/15384047.2018.1491501

35. Yewale, C., Baradia, D., Vhora, I., Patil, S., \& Misra, A. (2013). Epidermal growth factor receptor targeting in cancer: a review of trends and strategies. Biomaterials, 34(34), 8690-8707. doi:10.1016/j.biomaterials.2013.07.100

36. You, R., Hua, Y.-J., Liu, Y.-P., Yang, Q., Zhang, Y.-N., Li, J.-B., . . Chen, M.-Y. (2017). Concurrent Chemoradiotherapy with or without Anti-EGFR-Targeted Treatment for Stage II-IVb Nasopharyngeal Carcinoma: Retrospective Analysis with a Large Cohort and Long Follow-up. Theranostics, 7(8), 2314-2324. doi:10.7150/thno.19710

37. You, R., Sun, R., Hua, Y.-J., Li, C.-F., Li, J.-B., Zou, X., . . Chen, M.-Y. (2017). Cetuximab or nimotuzumab plus intensity-modulated radiotherapy versus cisplatin plus intensity-modulated radiotherapy for stage II-IVb nasopharyngeal carcinoma. International Journal of Cancer, 141(6), 1265-1276. doi:10.1002/ijc.30819 
38. Yu-Pei Chen, Nofisat Ismaila, Melvin L. K. Chua, A. Dimitrios Colevas, Robert Haddad, Shao Hui Huang, ... Ma., J. (2021). Chemotherapy in Combination With Radiotherapy for Definitive-Intent Treatment of Stage II-IVA Nasopharyngeal Carcinoma: CSCO and ASCO Guideline. Journal of Clinical Oncology, 39, 840-859. doi:10.1200/JC0.20

39. Zhang, M. X., Li, J., Shen, G. P., Zou, X., Xu, J. J., Jiang, R., . . Chen, M. Y. (2015). Intensity-modulated radiotherapy prolongs the survival of patients with nasopharyngeal carcinoma compared with conventional two-dimensional radiotherapy: A 10-year experience with a large cohort and long follow-up. Eur J Cancer, 51(17), 2587-2595. doi:10.1016/j.ejca.2015.08.006

40. Zhang, Y., Li, W. F., Liu, X., Chen, L., Sun, R., Sun, Y., . . Ma, J. (2018). Nomogram to predict the benefit of additional induction chemotherapy to concurrent chemoradiotherapy in locoregionally advanced nasopharyngeal carcinoma: Analysis of a multicenter, phase III randomized trial. Radiother Oncol, 129(1), 18-22. doi:10.1016/j.radonc.2017.12.002

41. Zhang, Z. C., Fu, S., Wang, F., Wang, H. Y., Zeng, Y. X., \& Shao, J. Y. (2014). Oncogene mutational profile in nasopharyngeal carcinoma. Onco Targets Ther, 7, 457-467. doi:10.2147/0TT.S58791

42. Zhi-gang Liu, Yu Zhao, Jiao Tang, Yu-juan Zhou, Wen-juan Yang, Yan-fang Qiu, \& Wang, H. (2016). Nimotuzumab combined with concurrent chemoradiotherapy in locally advanced nasopharyngeal carcinoma: a retrospective analysis. Oncotarget, 7.

43. Zhi-Qiang, W., Qi, M., Ji-Bin, L., Rui, Y., You-Ping, L., Rui, S., . . Yi-Jun, H. (2019). The long-term survival of patients with III-IVb stage nasopharyngeal carcinoma treated with IMRT with or without Nimotuzumab: a propensity score-matched analysis. BMC Cancer, 19(1). doi:10.1186/s12885-019$6156-5$

\section{Figures}

\section{Figure 1}

Diagram of the analytic cohort for survival analysis. Abbreviations: NPC, nasopharyngeal carcinoma; CCRT, concurrent chemoradiotherapy; SYSUCC, Sun Yat-Sen University Cancer Center; NTZ, nimotuzumab.

\section{Figure 2}

Kaplan-Meier survival curves based on CCRT plus nimotuzumab treatment versus CCRT alone for overall survival (a), progression-free survival (b), regional relapse-free survival (c), distant metastasis-free survival (d). Abbreviations: CCRT, concurrent chemoradiotherapy; NTZ, nimotuzumab. OS, Overall 
survival; PFS, progression-free survival; LRFS, locoregional recurrence-free survival; DMFS, distant metastasis-free survival.

\section{Figure 3}

Forest plot presented the univariate Cox regression analysis of prognostic factors influencing NPC patients' survival after propensity score matching.

\section{Figure 4}

Forest plot suggested the multivariate Cox regression analysis of prognostic factors influencing NPC patients' survival after propensity score matching.

\section{Figure 5}

Nomogram for 1-, 3- and 5-year OS (a), PFS (b), LRFS (c), and DMFS (d) in patients with nasopharyngeal carcinoma. The nomogram allows the user to obtain the probability of 1-,3- and 5-year survival based on a patient's combination of covariates. For example, the patient's treatment regime is identified, and a line is drawn straight upward to the "Points" axis to determine the score associated with that nimotuzumab. The process is repeated for each variable, the scores are summed and determined on the "Total Points" axis. A line is drawn straight down to determine the likelihood of 1-, 3- and 5-year survival. EBV DNA, Epstein-Barr virus DNA; OS, Overall survival; PFS, progression-free survival; LRFS, locoregional recurrence-free survival; DMFS, distant metastasis-free survival.

\section{Figure 6}

Calibration curve of nomogram models for predicting 1-, 3- and 5-year OS (a), PFS (b), LRFS (c), and DMFS (d). The actual survival is plotted on the y-axis; the nomogram predicts the probability of OS (a), PFS (b), LRFS (c), and DMFS (d), which is plotted on the x-axis, respectively. OS, Overall survival; PFS, progression-free survival; LRFS, locoregional recurrence-free survival; DMFS, distant metastasis-free survival.

\section{Supplementary Files}

This is a list of supplementary files associated with this preprint. Click to download. 
- SupplementalTable1.docx

- SupplementalTable2.docx 